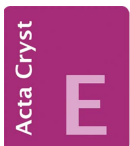

CRYSTALLOGRAPHIC COMMUNICATIONS

ISSN 2056-9890
Received 14 October 2021

Accepted 17 January 2022

Edited by M. Weil, Vienna University of Technology, Austria

Keywords: coordination compounds; iron(II); clathrate; platinum; cyanido ligand; crystal structure.

CCDC reference: 2142647

Supporting information: this article has supporting information at journals.iucr.org/e

\section{Crystal structure of poly[[diaquatetra- $\mu_{2}$-cyanido- platinum(II)iron(II)] methanol 4/3-solvate]: a three- dimensional Hofmann clathrate analogue}

\author{
Volodymyr M. Hiiuk, ${ }^{\text {a,b,c }}$ Vasyl Mykhailovych, ${ }^{\text {d }}$ Sergiu Shova, ${ }^{e}$ Irina A. Golenya ${ }^{a}$ \\ and Il'ya A. Gural'skiy ${ }^{a, b_{*}}$
}

a Department of Chemistry, Taras Shevchenko National University of Kyiv, 64 Volodymyrska St, 01601 Kyiv, Ukraine,
${ }^{\mathbf{b}}$ UkrOrgSyntez Ltd, 67 Chervonotkatska St, 02094 Kyiv, Ukraine, ' Faculty of Natural Sciences, National University of
Kyiv-Mohyla Academy, 2 Skovorody St, 04070 Kyiv, Ukraine, ${ }^{\mathbf{d}}$ Faculty of Electrical Engineering and Computer Science \&
Research Center, MANSiD, Stefan cel Mare University, 13 Universitatii St., 720229 Suceava, Romania, and ${ }^{\mathbf{e}}$ Department
of Inorganic Polymers, "Petru Poni", Institute of Macromolecular Chemistry, Romanian Academy of Science, Aleea
Grigore Ghica Voda 41-A, lasi 700487, Romania. *Correspondence e-mail: igolenya@ua.fm

In the title polymeric coordination compound, $\left\{\left[\mathrm{FePt}(\mathrm{CN})_{4}\left(\mathrm{H}_{2} \mathrm{O}\right)_{2}\right] \cdot-\right.$ $\left.1.33 \mathrm{CH}_{3} \mathrm{OH}\right\}_{n}$, the $\mathrm{Fe}^{\mathrm{II}}$ cation (site symmetry $4 / \mathrm{mm} . \mathrm{m}$ ) is coordinated by the $\mathrm{N}$ atoms of four cyanide anions $\left(\mathrm{CN}^{-}\right)$and the $\mathrm{O}$ atoms of two water molecules, forming a nearly regular $\left[\mathrm{FeN}_{4} \mathrm{O}_{2}\right]$ octahedron. According the $\mathrm{Fe}-\mathrm{N}$ and $\mathrm{Fe}-\mathrm{O}$ bond lengths, the $\mathrm{Fe}^{\mathrm{II}}$ atom is in the high-spin state. The cyanide anions act in a bridging manner to connect the $\mathrm{Fe}^{\mathrm{II}}$ and $\mathrm{Pt}^{\mathrm{II}}$ atoms. The $\left[\mathrm{Pt}(\mathrm{CN})_{4}\right]^{2-}$ moieties $(\mathrm{Pt}$ with site symmetry $4 / \mathrm{mm} . \mathrm{m}$ ) have a perfect square-planar shape. The latter anion is located perpendicular to the $\mathrm{FeN}_{4}$ plane, thus ensuring the creation of a three-dimensional framework. The crystal structure features methanol solvent molecules of which $4 / 3$ were located per $\mathrm{Fe}^{\mathrm{II}}$ cation. These solvent molecules are located in hexagonal pores; they interact with coordinating water molecules through weak hydrogen bonds. Other guest molecules could not be modelled in a satisfactory way and their contribution to the scattering was removed by a mask procedure.

\section{Chemical context}

Cyanide-based complexes form a large group of coordination compounds, which can offer numerous structures and functionalities. As a result of the ability of the cyanide anion to act in a bridging way, this group often links two different metal cations, enabling the formation of one-, two- or threedimensional frameworks. The beginning of the investigation of cyanide-based complexes dates back to the 18th century when Prussian blue was discovered (Dacarro et al., 2018). Since then, hundreds of cyanide-based complexes have been obtained and proven to be efficient as molecular magnets, in separation, condensation, storage, catalysis, polymer synthesis, switching, etc (Zakaria \& Chikyow, 2017).

Among all cyanide-based complexes, Hofmann clathrate analogues attract considerable attention. This is a group of polymeric coordination complexes with general formula $\left[M(L)_{x}\left\{M^{\prime}(\mathrm{CN})_{y}\right\}_{z} \cdot n\right.$ (guest/solvent) where $M$ has an octahedral coordination environment with two $L$ ligands in axial positions and four $\mathrm{N}$ atoms of bridging cyanide groups in equatorial positions, which link $M$ and $M^{\prime}$ metals into infinite layers (Powell \& Rayner, 1949; Hofmann \& Küspert, 1897). If the $L$ ligand is bridging as well (e.g. pyrazine), the creation of a 
three-dimensional framework is observed (Niel et al., 2001). In addition, the chemical composition of Hofmann clathrates can easily be modified by variation of the guest/solvent molecules.

One of the attractive properties of Hofmann clathrate analogues is the ability of some complexes of this class to undergo spin crossover under the influence of external stimuli (Carmen Muñoz \& Real, 2011; Kucheriv et al., 2021). The change of spin state can be observed in complexes of general formula $\left[\mathrm{Fe}(L)_{x}\left\{M^{\prime}(\mathrm{CN})_{y}\right\}_{z}\right]$ where $L=$ azine or azole ligand, $M^{\prime}=\mathrm{Cu}, \mathrm{Ag}, \mathrm{Au}$ for $y=2, z=2$, and $M^{\prime}=\mathrm{Ni}, \mathrm{Pt}, \mathrm{Pd}$ for $y=4$, $z=1$ (Shylin et al., 2020; Kuzevanova et al., 2021).

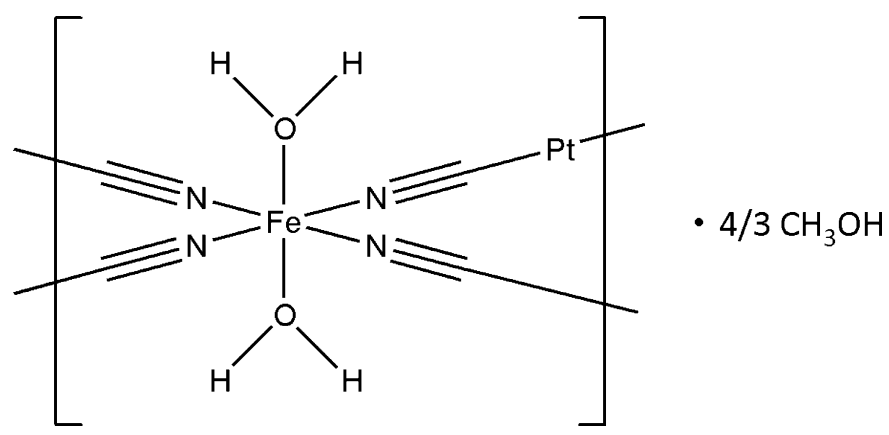

In this paper we report a $\left\{\left[\mathrm{FePt}(\mathrm{CN})_{4}\left(\mathrm{H}_{2} \mathrm{O}\right)_{2}\right] \cdot 4 / 3 \mathrm{CH}_{3} \mathrm{OH}\right\}_{n}$ coordination polymer with a non-classical Hofmann-type framework.

\section{Structural commentary}

The $\mathrm{Fe}^{\mathrm{II}}$ cation (site symmetry 4/mm.m; Wyckoff position $3 c$ ) exists in an $\left[\mathrm{FeN}_{4} \mathrm{O}_{2}\right]$ coordination environment (Fig. 1), which is formed by the $\mathrm{N}$ atoms of four cyanide anions in equatorial positions $[\mathrm{Fe} 1-\mathrm{N} 1=2.155(18) \AA]$ and the $\mathrm{O}$ atoms of two water molecules in axial positions $[\mathrm{Fe} 1-\mathrm{O} 1=2.15$ (2) $\AA$ ] The similar lengths of the $\mathrm{Fe}-\mathrm{O}$ and $\mathrm{Fe}-\mathrm{N}$ bonds provide an almost ideal octahedral environment. The $\mathrm{Fe}^{\mathrm{II}}-\mathrm{O}$ and $\mathrm{Fe}^{\mathrm{II}}-$ $\mathrm{N}$ bond lengths indicate that, at the temperature of the

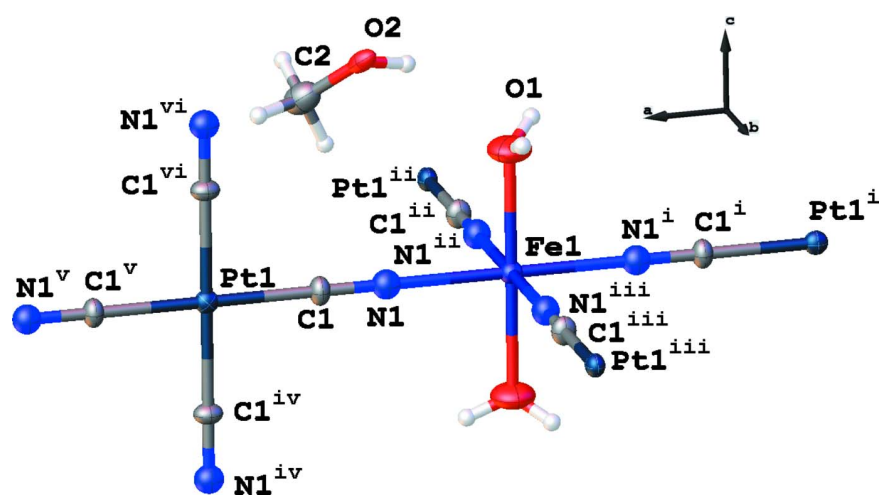

Figure 1

A fragment of the crystal structure of the title compound showing the atom-labelling scheme. Displacement ellipsoids are drawn at the $50 \%$ probability level. [Symmetry codes: (i) $1-x, 1-y,+z$; (ii) $y, 1-x,+z$; (iii) $1-y, x, z$; (iv) $1-z, y,-1+x$; (v) $2-x, 1-y, z$; (vi) $1+z, y, 1-x$; (vii) $x, 1-y,-z]$.
Table 1

Hydrogen-bond geometry $\left(\AA{ }^{\circ}\right)$.

\begin{tabular}{lllll}
\hline$D-\mathrm{H} \cdots A$ & $D-\mathrm{H}$ & $\mathrm{H} \cdots A$ & $D \cdots A$ & $D-\mathrm{H} \cdots A$ \\
\hline $\mathrm{O} 1-\mathrm{H} 1 A \cdots \mathrm{O} 2$ & 0.82 & 2.20 & $3.020(18)$ & 175 \\
\hline
\end{tabular}

diffraction study, $\mathrm{Fe}^{\mathrm{II}}$ is in the high-spin state. The cyanide anions connect the $\mathrm{Fe}^{\mathrm{II}}$ and $\mathrm{Pt}^{\mathrm{II}}$ atoms, whereby the latter (site symmetry $4 / m m . m$; Wyckoff position $3 d$ ) has a perfect squareplanar environment with a Pt1-C1 bond length of 1.953 (17) A. Contrary to classical Hofmann clathrate arrangements (Kucheriv et al., 2021), the tetracyanidoplatinate(II) anions in the title compound are located perpendicular to the $\mathrm{FeN}_{4}$ plane, which ensures the creation of a three-dimensional framework (Fig. 2). As a result of the cubic symmetry of the crystal structure, no deviation from linearity is observed for the $\mathrm{Fe}-\mathrm{N}-\mathrm{C}-\mathrm{Pt}$ fragments.

The title compound incorporates $4 / 3$ methanol solvent molecules per $\left[\mathrm{FePt}(\mathrm{CN})_{4}\left(\mathrm{H}_{2} \mathrm{O}\right)_{2}\right]$ unit, which are located in hexagonal pores (Fig. 3) and interact with the coordinating water molecules through $\mathrm{O}-\mathrm{H} \cdots \mathrm{O}$ hydrogen bonds (Table 1). The framework features some additional highly disordered guest molecules, which could not be modelled satisfactorily. Their contribution to the scattering was removed with a mask procedure implemented in OLEX2 (Dolomanov et al., 2009). These disordered guest molecules reside in two types of void with total volumes of 138.3 and $20.3 \AA^{3}$ corresponding to 36.4 and 2.6 electrons, respectively.

In comparison, two similar coordination compounds, viz. $\left[\mathrm{Fe}\left(\mathrm{H}_{2} \mathrm{O}\right)_{2}\left\{\mathrm{Pt}(\mathrm{CN})_{4}\right] \cdot 2\right.$ acetone (Kuzevanova et al., 2019) and $\left[\mathrm{Fe}\left(\mathrm{H}_{2} \mathrm{O}\right)_{2}\left\{\mathrm{Ni}(\mathrm{CN})_{4}\right\}\right] \cdot 2$ dioxane (Yuge et al., 1997), form infinite $\left\{\mathrm{Fe} M^{\mathrm{II}}(\mathrm{CN})_{4}\right\}_{\infty}$ layers. The size of the available voids between the cyanidometallate layers in these two compounds allows the acetone or dioxane molecules to rotate freely, thus leading to a high disorder of the solvent. Both of these compounds, as well as the title compound, represent specta-

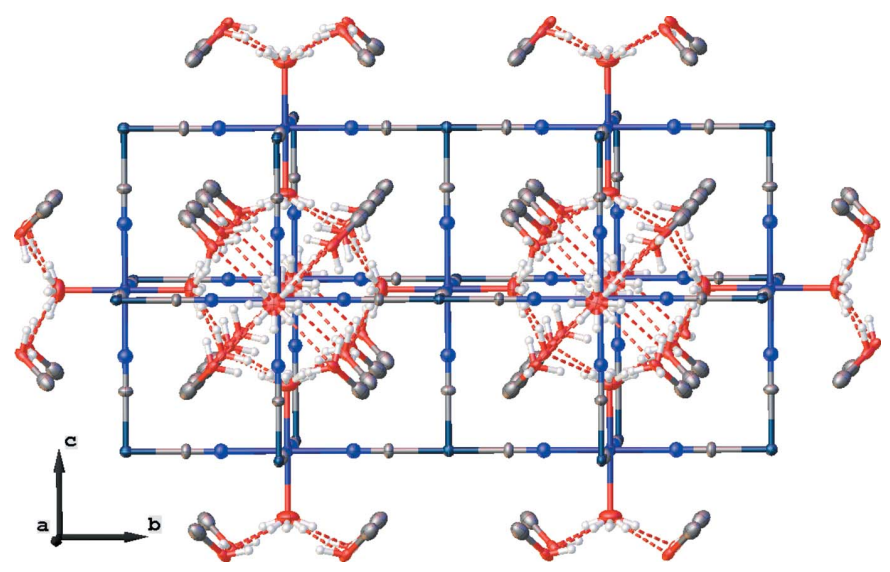

Figure 2

View of the crystal structure of the title compound along the $a$ axis showing the three-dimensional coordination framework. Hydrogen bonds are shown as red dashed lines. Hydrogen atoms of the methyl group of the methanol solvent molecules are omitted for clarity. 


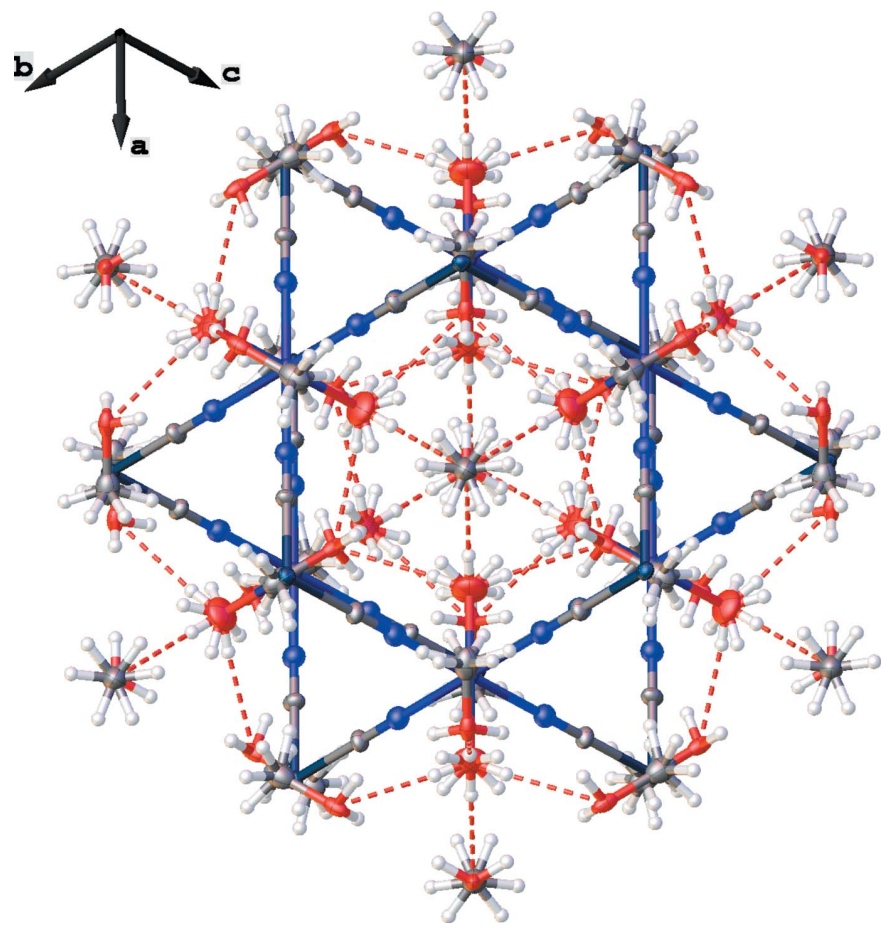

Figure 3

View of the crystal structure of the title compound showing the methanol solvent molecules, which are located in hexagonal pores. Hydrogen bonds are shown as red dashed lines.

cular examples of how variation of the guest/solvent molecule can significantly influence the crystal structure of the coordination framework. Whereas small molecules of methanol can fit inside the hexagonal pores of a three-dimensional framework, bulkier acetone or dioxane molecules cannot be placed there, thus inducing the creation of layers.

\section{Database survey}

A survey of the Cambridge Structural Database (Version 5.40; Groom et al., 2016) revealed 106 framework structures containing $\mathrm{Fe}-\mathrm{N}-\mathrm{C}-\mathrm{Pt}$ fragments. Among them there are three structures with an $\left[\mathrm{FeN}_{5} \mathrm{O}\right]$ coordination environment [AMIJEN (Kucheriv et al., 2016), ZOHBEG and ZOHBIK (Wong et al., 2019)], three structures with an $\left[\mathrm{FeN}_{4} \mathrm{O}_{2}\right]$ coordination environment [CEMJAI (Piñeiro-López et al., 2017), HOCRAU (Zhang et al., 2014) and OKITAF (Haraguchi et al., 2016)] and three structures that have two different $\mathrm{Fe}^{\mathrm{II}}$ cations forming $\left[\mathrm{FeN}_{4} \mathrm{O}_{2}\right]$ and $\left[\mathrm{FeN}_{6}\right]$ octahedra [AMIJOX (Kucheriv et al., 2016) and VOKLIS, VOKLIS01 (Sciortino et al., 2014)].

\section{Synthesis and crystallization}

Crystals of the title compound were grown by slow diffusion between three layers in a $3 \mathrm{ml}$ tube. The first layer was a solution of $\mathrm{K}_{2}\left[\mathrm{Pt}(\mathrm{CN})_{4}\right](0.02 \mathrm{mmol})$ in water $(0.5 \mathrm{ml})$, the second was a mixture of water/methanol $(1: 1,1.5 \mathrm{ml})$ and the third layer was a solution of $\mathrm{Fe}(\mathrm{OTs})_{2} \cdot 6 \mathrm{H}_{2} \mathrm{O}(0.02 \mathrm{mmol})$
Table 2

Experimental details.

\begin{tabular}{|c|c|}
\hline \multicolumn{2}{|l|}{ Crystal data } \\
\hline Chemical formula & {$\left[\mathrm{FePt}(\mathrm{CN})_{4}\left(\mathrm{H}_{2} \mathrm{O}\right)_{2}\right] \cdot 1.33 \mathrm{CH}_{4} \mathrm{O}$} \\
\hline$M_{\mathrm{r}}$ & 433.77 \\
\hline Crystal system, space group & Cubic, $P m \overline{3} m$ \\
\hline Temperature $(\mathrm{K})$ & 293 \\
\hline$a(\AA)$ & $10.5089(3)$ \\
\hline$V\left(\AA^{3}\right)$ & $1160.56(10)$ \\
\hline$Z$ & 3 \\
\hline Radiation type & Мo $K \alpha$ \\
\hline$\mu\left(\mathrm{mm}^{-1}\right)$ & 9.96 \\
\hline Crystal size $(\mathrm{mm})$ & $0.04 \times 0.04 \times 0.04$ \\
\hline \multicolumn{2}{|l|}{ Data collection } \\
\hline Diffractometer & Xcalibur, Eos \\
\hline Absorption correction & $\begin{array}{l}\text { Multi-scan (CrysAlis PRO; Rigaku } \\
\text { OD, 2021) }\end{array}$ \\
\hline$T_{\min }, T_{\max }$ & $0.930,1.000$ \\
\hline $\begin{array}{l}\text { No. of measured, independent and } \\
\text { observed }[I>2 \sigma(I)] \text { reflections }\end{array}$ & $2210,336,273$ \\
\hline$R_{\text {int }}$ & 0.091 \\
\hline$(\sin \theta / \lambda)_{\max }\left(\AA^{-1}\right)$ & 0.688 \\
\hline \multicolumn{2}{|l|}{ Refinement } \\
\hline$R\left[F^{2}>2 \sigma\left(F^{2}\right)\right], w R\left(F^{2}\right), S$ & $0.055,0.133,1.07$ \\
\hline No. of reflections & 336 \\
\hline No. of parameters & 23 \\
\hline No. of restraints & 13 \\
\hline $\mathrm{H}$-atom treatment & $\mathrm{H}$-atom parameters constrained \\
\hline$\Delta \rho_{\max }, \Delta \rho_{\min }\left(\mathrm{e} \AA^{-3}\right)$ & $2.15,-1.36$ \\
\hline
\end{tabular}

Computer programs: CrysAlis PRO (Rigaku OD, 2021), SHELXT (Sheldrick, 2015a), SHELXL (Sheldrick, 2015b) and OLEX2 (Dolomanov et al., 2009).

(OTs $=p$-toluenesulfonate) in methanol $(0.5 \mathrm{ml})$. After two weeks, colourless crystals grew in the middle layer; these were collected and maintained under the mother solution until measured.

\section{Refinement}

Crystal data, data collection and structure refinement details are summarized in Table $2 . \mathrm{H}$ atoms of water molecules and the methanol $\mathrm{OH}$ group were placed at calculated positions and refined as riding on the bonded $\mathrm{O}$ atom. The occupancy of methanol atoms was refined and found to be equal to approximately 0.5 and later restrained to half-occupancy. As a result of symmetry restrictions, $\mathrm{H}$ atoms of the water molecule are disordered over four positions and were constrained to have an occupancy of $1 / 4$. The three $\mathrm{H}$ atoms of the methyl group are disordered over two sets of sites, and were refined as for an idealized methyl group and were allowed to rotate about the $\mathrm{O}-\mathrm{C}$ bond. The $\mathrm{H}$ atom of the $\mathrm{OH}$ group is disordered over three sites. Its occupancy was restrained to coincide with half-occupancy of the complete molecule.

\section{Funding information}

Funding for this research was provided by: Ministry of Education and Science of Ukraine (grant No. 19BF037-01M); H2020 Marie Skłodowska-Curie Actions (grant No. 734322). VMH thanks the Yuchymenko Family Endowment Fund for financial support. 


\section{References}

Carmen Muñoz, M. \& Real, J. A. (2011). Coord. Chem. Rev. 255, 2068-2093.

Dacarro, G., Taglietti, A. \& Pallavicini, P. (2018). Molecules, 23, 1414.

Dolomanov, O. V., Bourhis, L. J., Gildea, R. J., Howard, J. A. K. \& Puschmann, H. (2009). J. Appl. Cryst. 42, 339-341.

Groom, C. R., Bruno, I. J., Lightfoot, M. P. \& Ward, S. C. (2016). Acta Cryst. B72, 171-179.

Haraguchi, T., Otsubo, K., Sakata, O., Kawaguchi, S., Fujiwara, A. \& Kitagawa, H. (2016). Chem. Commun. 52, 6017-6020.

Hofmann, K. A. \& Küspert, F. (1897). Z. Anorg. Chem. 15, 204-207.

Kucheriv, O. I., Fritsky, I. O. \& Gural'skiy, I. A. (2021). Inorg. Chim. Acta, 521, 120303.

Kucheriv, O. I., Shylin, S. I., Ksenofontov, V., Dechert, S., Haukka, M., Fritsky, I. O. \& Gural'skiy, I. A. (2016). Inorg. Chem. 55, 4906-4914.

Kuzevanova, I. S., Kucheriv, O. I., Hiiuk, V. M., Naumova, D. D., Shova, S., Shylin, S. I., Kotsyubynsky, V. O., Rotaru, A., Fritsky, I. O. \& Gural'skiy, I. A. (2021). Dalton Trans. 50, 9250-9258.

Kuzevanova, I. S., Naumova, D. D., Terebilenko, K. V., Shova, S. \& Gural'skiy, I. A. (2019). Acta Cryst. E75, 1536-1539.
Niel, V., Martinez-Agudo, J. M., Carmen Muñoz, M., Gaspar, A. B. \& Real, J. A. (2001). Inorg. Chem. 40, 3838-3839.

Piñeiro-López, L., Valverde-Muñoz, F. J., Seredyuk, M. \& Znovjyak, K. (2017). IUCrData, 2, x171413.

Powell, H. M. \& Rayner, J. H. (1949). Nature, 163, 566-567.

Rigaku OD (2021). CrysAlis PRO. Rigaku Oxford Diffraction, Yarnton, England.

Sciortino, N. F., Neville, S. M., Létard, J.-F., Moubaraki, B., Murray, K. S. \& Kepert, C. J. (2014). Inorg. Chem. 53, 7886-7893.

Sheldrick, G. M. (2015a). Acta Cryst. A71, 3-8.

Sheldrick, G. M. (2015b). Acta Cryst. C71, 3-8.

Shylin, S. I., Kucheriv, O. I., Shova, S., Ksenofontov, V., Tremel, W. \& Gural'skiy, I. A. (2020). Inorg. Chem. 59, 6541-6549.

Wong, B. J. C., Xu, D., Bao, S.-S., Zheng, L.-M. \& Lei, J. (2019). Appl. Mater. Interfaces, 11, 12986-12992.

Yuge, H., Kim, C., Iwamoto, T. \& Kitazawa, T. (1997). Inorg. Chim. Acta, 257, 217-224.

Zakaria, M. B. \& Chikyow, T. (2017). Coord. Chem. Rev. 352, 328345.

Zhang, M., Li, B.-B., Sun, J., Kong, X.-P., Gu, P.-P., Chen, Y.-Y. \& Yuan, A.-H. (2014). Z. Anorg. Allg. Chem. 640, 1007-1011. 


\section{supporting information}

Acta Cryst. (2022). E78, 216-219 [https://doi.org/10.1107/S2056989022000573]

Crystal structure of poly[[diaquatetra- $\mu_{2}$-cyanido-platinum(II)iron(II)] methanol 4/3-solvate]: a three-dimensional Hofmann clathrate analogue

Volodymyr M. Hiiuk, Vasyl Mykhailovych, Sergiu Shova, Irina A. Golenya and II'ya A.

\section{Gural'skiy}

Computing details

Data collection: CrysAlis PRO (Rigaku OD, 2021); cell refinement: CrysAlis PRO (Rigaku OD, 2021); data reduction: CrysAlis PRO (Rigaku OD, 2021); program(s) used to solve structure: SHELXT (Sheldrick, 2015a); program(s) used to refine structure: SHELXL (Sheldrick, 2015b); molecular graphics: OLEX2 (Dolomanov et al., 2009); software used to prepare material for publication: OLEX2 (Dolomanov et al., 2009).

Poly[[diaquatetra- $\mu_{2}$-cyanido-platinum(II)iron(II)] methanol 1.33-solvate]

Crystal data

$\left[\mathrm{FePt}(\mathrm{CN})_{4}\left(\mathrm{H}_{2} \mathrm{O}\right)_{2}\right] \cdot 1.33 \mathrm{CH}_{4} \mathrm{O}$

$M_{r}=433.77$

Cubic, $P m \overline{3} m$

$a=10.5089(3) \AA$

$V=1160.56(10) \AA^{3}$

$Z=3$

$F(000)=600$

$D_{\mathrm{x}}=1.862 \mathrm{Mg} \mathrm{m}^{-3}$

Data collection

Xcalibur, Eos diffractometer

Radiation source: fine-focus sealed X-ray tube, Enhance (Mo) X-ray Source

Graphite monochromator

Detector resolution: 16.1593 pixels $\mathrm{mm}^{-1}$

$\omega$ scans

Absorption correction: multi-scan

(CrysAlisPro; Rigaku OD, 2021)

Refinement

Refinement on $F^{2}$

Least-squares matrix: full

$R\left[F^{2}>2 \sigma\left(F^{2}\right)\right]=0.055$

$w R\left(F^{2}\right)=0.133$

$S=1.07$

336 reflections

23 parameters

13 restraints
Mo $K \alpha$ radiation, $\lambda=0.71073 \AA$

Cell parameters from 449 reflections

$\theta=1.9-21.7^{\circ}$

$\mu=9.96 \mathrm{~mm}^{-1}$

$T=293 \mathrm{~K}$

Cube, clear intense colourless

$0.04 \times 0.04 \times 0.04 \mathrm{~mm}$

$T_{\min }=0.930, T_{\max }=1.000$

2210 measured reflections

336 independent reflections

273 reflections with $I>2 \sigma(I)$

$R_{\text {int }}=0.091$

$\theta_{\text {max }}=29.3^{\circ}, \theta_{\text {min }}=1.9^{\circ}$

$h=-9 \rightarrow 8$

$k=-14 \rightarrow 14$

$l=-5 \rightarrow 14$

Hydrogen site location: mixed

$\mathrm{H}$-atom parameters constrained $w=1 /\left[\sigma^{2}\left(F_{\mathrm{o}}{ }^{2}\right)+(0.0611 P)^{2}\right]$

where $P=\left(F_{\mathrm{o}}{ }^{2}+2 F_{\mathrm{c}}{ }^{2}\right) / 3$

$(\Delta / \sigma)_{\max }<0.001$

$\Delta \rho_{\max }=2.15 \mathrm{e}^{-3}$

$\Delta \rho_{\min }=-1.36$ e $\AA^{-3}$ 


\section{Special details}

Geometry. All esds (except the esd in the dihedral angle between two 1.s. planes) are estimated using the full covariance matrix. The cell esds are taken into account individually in the estimation of esds in distances, angles and torsion angles; correlations between esds in cell parameters are only used when they are defined by crystal symmetry. An approximate (isotropic) treatment of cell esds is used for estimating esds involving l.s. planes.

Fractional atomic coordinates and isotropic or equivalent isotropic displacement parameters $\left(\AA^{2}\right)$

\begin{tabular}{llllll}
\hline & $x$ & $y$ & $z$ & $U_{\text {iso }} / U_{\text {eq }}$ & Occ. $(<1)$ \\
\hline Pt1 & 1.000000 & 0.500000 & 0.000000 & $0.0374(5)$ & \\
Fe1 & 0.500000 & 0.500000 & 0.000000 & $0.0395(12)$ & \\
N1 & $0.7051(17)$ & 0.500000 & 0.000000 & $0.059(4)$ & \\
C1 & $0.8141(17)$ & 0.500000 & 0.000000 & $0.047(4)$ & \\
O1 & 0.500000 & 0.500000 & $0.2049(19)$ & $0.112(9)$ & \\
H1A & 0.549690 & 0.450310 & 0.238738 & $0.168^{*}$ & 0.25 \\
H1B & 0.423680 & 0.500000 & 0.233848 & $0.168^{*}$ & 0.25 \\
O2 & $0.6888(17)$ & $0.3112(17)$ & $0.3112(17)$ & $0.049(7)$ & 0.5 \\
H2 & 0.610990 & 0.294000 & 0.294000 & $0.073^{*}$ & 0.1667 \\
C2 & $0.7666(19)$ & $0.2334(19)$ & $0.2334(19)$ & $0.088(14)$ & 0.5 \\
H2A & 0.728153 & 0.224628 & 0.151003 & $0.132^{*}$ & 0.0833 \\
H2B & 0.775372 & 0.151003 & 0.271847 & $0.132^{*}$ & 0.0833 \\
H2C & 0.848997 & 0.271847 & 0.224628 & $0.132^{*}$ & 0.0833 \\
& & & & & \\
\hline
\end{tabular}

Atomic displacement parameters $\left(\AA^{2}\right)$

\begin{tabular}{lllllll}
\hline & $U^{11}$ & $U^{22}$ & $U^{33}$ & $U^{12}$ & $U^{13}$ & $U^{23}$ \\
\hline Pt1 & $0.0420(6)$ & $0.0282(7)$ & $0.0420(6)$ & 0.000 & 0.000 & 0.000 \\
Fe1 & $0.0399(16)$ & $0.0399(16)$ & $0.039(2)$ & 0.000 & 0.000 & 0.000 \\
N1 & $0.060(11)$ & $0.054(10)$ & $0.064(11)$ & 0.000 & 0.000 & 0.000 \\
C1 & $0.032(9)$ & $0.046(10)$ & $0.063(12)$ & 0.000 & 0.000 & 0.000 \\
O1 & $0.144(15)$ & $0.144(15)$ & $0.047(14)$ & 0.000 & 0.000 & 0.000 \\
O2 & $0.049(7)$ & $0.049(7)$ & $0.049(7)$ & $-0.019(7)$ & $-0.019(7)$ & $0.019(7)$ \\
C2 & $0.088(14)$ & $0.088(14)$ & $0.088(14)$ & $-0.021(11)$ & $-0.021(11)$ & $0.021(11)$ \\
\hline
\end{tabular}

Geometric parameters $\left(A,{ }^{\circ}\right)$

\begin{tabular}{llll}
\hline $\mathrm{Pt} 1-\mathrm{C} 1^{\mathrm{i}}$ & $1.953(17)$ & $\mathrm{O} 1-\mathrm{H} 1 \mathrm{~A}$ & 0.8198 \\
$\mathrm{Pt} 1-\mathrm{C} 1^{1 i}$ & $1.953(17)$ & $\mathrm{O} 1-\mathrm{H}^{\mathrm{ii}}$ & 0.8198 \\
$\mathrm{Pt} 1-\mathrm{C} 1^{\text {iii }}$ & $1.953(17)$ & $\mathrm{O} 1-\mathrm{H}^{\mathrm{iix}}$ & 0.8579 \\
$\mathrm{Pt} 1-\mathrm{C} 1$ & $1.953(17)$ & $\mathrm{O} 1-\mathrm{H}^{\mathrm{ix}}$ & 0.8579 \\
$\mathrm{Fe} 1-\mathrm{N} 1$ & $2.155(18)$ & $\mathrm{O} 1-\mathrm{H} 1 \mathrm{~B}^{\mathrm{viii}}$ & 0.8579 \\
$\mathrm{Fe} 1-\mathrm{N} 1^{\mathrm{iv}}$ & $2.155(18)$ & $\mathrm{O} 1-\mathrm{H} 1 \mathrm{~B}$ & 0.8579 \\
$\mathrm{Fe} 1-\mathrm{N} 1^{\mathrm{v}}$ & $2.155(18)$ & $\mathrm{O} 2-\mathrm{H} 2$ & 0.8565 \\
$\mathrm{Fe} 1-\mathrm{N} 1^{\mathrm{vi}}$ & $2.155(18)$ & $\mathrm{O} 2-\mathrm{H} 2^{\mathrm{xi}}$ & 0.8565 \\
$\mathrm{Fe} 1-\mathrm{O} 1^{\mathrm{v}}$ & $2.15(2)$ & $\mathrm{O} 2-\mathrm{H} 2^{\mathrm{xii}}$ & 0.8565 \\
$\mathrm{Fe} 1-\mathrm{O} 1$ & $2.15(2)$ & $\mathrm{O} 2-\mathrm{C} 2$ & $1.42(2)$ \\
$\mathrm{N} 1-\mathrm{C} 1$ & $1.15(2)$ & $\mathrm{C} 2-\mathrm{H} 2 \mathrm{~A}$ & 0.9600 \\
$\mathrm{O} 1-\mathrm{H} 1 \mathrm{~A}^{\mathrm{vii}}$ & 0.8198 & $\mathrm{C} 2-\mathrm{H} 2 \mathrm{~B}$ & 0.9600
\end{tabular}




\begin{tabular}{|c|c|c|c|}
\hline $\mathrm{O} 1-\mathrm{H} 1 \mathrm{~A}^{\mathrm{viii}}$ & 0.8198 & $\mathrm{C} 2-\mathrm{H} 2 \mathrm{C}$ & 0.9600 \\
\hline $\mathrm{C} 1^{\mathrm{i}}-\mathrm{Pt} 1-\mathrm{C} 1^{\mathrm{iii}}$ & 180.0 & $\mathrm{H} 1 \mathrm{~A}-\mathrm{O} 1-\mathrm{H} 1 \mathrm{~A}^{\mathrm{ix}}$ & 79.1 \\
\hline $\mathrm{C} 11^{\mathrm{iii}}-\mathrm{Pt} 1-\mathrm{C} 1$ & 90.0 & $\mathrm{H} 1 \mathrm{~A}^{\mathrm{viii}}-\mathrm{O} 1-\mathrm{H} 1 \mathrm{~B}^{\mathrm{x}}$ & 116.2 \\
\hline $\mathrm{C} 1 \mathrm{i}-\mathrm{Pt} 1-\mathrm{C} 1$ & 90.0 & $\mathrm{H} 1 \mathrm{~A}-\mathrm{O} 1-\mathrm{H}_{1} \mathrm{~B}^{\mathrm{x}}$ & 41.4 \\
\hline $\mathrm{C} 1^{\mathrm{i}}-\mathrm{Pt} 1-\mathrm{C} 1^{\mathrm{ii}}$ & 90.0 & $\mathrm{H} 1 \mathrm{~A}^{\mathrm{vii}}-\mathrm{O} 1-\mathrm{H}_{1} \mathrm{~B}^{\mathrm{viii}}$ & 116.2 \\
\hline $\mathrm{C} 1^{\mathrm{iii}}-\mathrm{Pt} 1-\mathrm{C} 1^{\mathrm{ii}}$ & 90.0 & $\mathrm{H} 1 \mathrm{~A}^{\mathrm{vii}}-\mathrm{O} 1-\mathrm{H}_{1} \mathrm{~B}^{\mathrm{ix}}$ & 41.4 \\
\hline $\mathrm{C} 1^{\mathrm{ii}}-\mathrm{Pt} 1-\mathrm{C} 1$ & 180.0 & $\mathrm{H} 1 \mathrm{~A}-\mathrm{O} 1-\mathrm{H} 1 \mathrm{~B}$ & 116.2 \\
\hline $\mathrm{N} 1-\mathrm{Fe} 1-\mathrm{N} 1^{\mathrm{v}}$ & 180.0 & $\mathrm{H} 1 \mathrm{~A}^{\mathrm{vii}}-\mathrm{O} 1-\mathrm{H}_{1 \mathrm{~B}^{\mathrm{x}}}$ & 41.4 \\
\hline 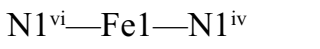 & 180.0 & $\mathrm{H} 1 \mathrm{~A}-\mathrm{O} 1-\mathrm{H} 1 \mathrm{~B}^{\mathrm{ix}}$ & 116.2 \\
\hline $\mathrm{N} 1^{\mathrm{v}}-\mathrm{Fe} 1-\mathrm{N} 1^{\mathrm{iv}}$ & 90.0 & $\mathrm{H} 1 \mathrm{~A}-\mathrm{O} 1-\mathrm{H}_{1} \mathrm{~B}^{\mathrm{viii}}$ & 41.4 \\
\hline $\mathrm{N} 1-\mathrm{Fe} 1-\mathrm{N} 1^{\mathrm{vi}}$ & 90.0 & $\mathrm{H} 1 \mathrm{~A}^{\mathrm{ix}}-\mathrm{O} 1-\mathrm{H}_{1} \mathrm{~B}^{\mathrm{x}}$ & 116.2 \\
\hline $\mathrm{N} 1^{\mathrm{v}}-\mathrm{Fe} 1-\mathrm{N} 1^{\mathrm{vi}}$ & 90.0 & $\mathrm{H} 1 \mathrm{~A}^{\mathrm{ix}}-\mathrm{O} 1-\mathrm{H}_{1} \mathrm{~B}^{\mathrm{viii}}$ & 41.4 \\
\hline $\mathrm{N} 1-\mathrm{Fe} 1-\mathrm{N} 1^{\mathrm{iv}}$ & 90.0 & $\mathrm{H} 1 \mathrm{~A}^{\mathrm{viii}}-\mathrm{O} 1-\mathrm{H} 1 \mathrm{~B}^{\mathrm{viii}}$ & 116.2 \\
\hline $\mathrm{O} 1^{\mathrm{v}}-\mathrm{Fe} 1-\mathrm{N} 1^{\mathrm{vi}}$ & 90.0 & $\mathrm{H} 1 \mathrm{~A}^{\mathrm{viii}}-\mathrm{O} 1-\mathrm{H}_{1} \mathrm{~B}^{\mathrm{ix}}$ & 41.4 \\
\hline $\mathrm{O} 1^{\mathrm{v}}-\mathrm{Fe} 1-\mathrm{N} 1^{\mathrm{iv}}$ & 90.0 & $\mathrm{H} 1 \mathrm{~A}^{\mathrm{ix}}-\mathrm{O} 1-\mathrm{H} 1 \mathrm{~B}^{\mathrm{ix}}$ & 116.2 \\
\hline $\mathrm{O} 1-\mathrm{Fe} 1-\mathrm{N} 1^{\mathrm{v}}$ & 90.0 & $\mathrm{H} 1 \mathrm{~B}-\mathrm{O} 1-\mathrm{H} 1 \mathrm{~A}^{\mathrm{vii}}$ & 116.2 \\
\hline $\mathrm{O} 1-\mathrm{Fe} 1-\mathrm{N} 1^{\mathrm{vi}}$ & 90.0 & $\mathrm{H} 1 \mathrm{~B}-\mathrm{O} 1-\mathrm{H}_{1} \mathrm{~A}^{\mathrm{ix}}$ & 41.4 \\
\hline $\mathrm{O} 1-\mathrm{Fe} 1-\mathrm{N} 1^{\mathrm{iv}}$ & 90.0 & $\mathrm{H} 1 \mathrm{~B}-\mathrm{O} 1-\mathrm{H} 1 \mathrm{~A}^{\mathrm{viii}}$ & 41.4 \\
\hline $\mathrm{O} 1^{\mathrm{v}}-\mathrm{Fe} 1-\mathrm{N} 1$ & 90.0 & $\mathrm{H} 1 \mathrm{~B}^{\mathrm{ix}}-\mathrm{O} 1-\mathrm{H}_{1} \mathrm{~B}^{\mathrm{viii}}$ & 138.4 \\
\hline $\mathrm{O} 1-\mathrm{Fe} 1-\mathrm{N} 1$ & 90.0 & $\mathrm{H} 1 \mathrm{~B}^{\mathrm{viii}}-\mathrm{O} 1-\mathrm{H} 1 \mathrm{~B}^{\mathrm{x}}$ & 82.8 \\
\hline $\mathrm{O} 1^{\mathrm{v}}-\mathrm{Fe} 1-\mathrm{N} 1^{\mathrm{v}}$ & 90.0 & $\mathrm{H} 1 \mathrm{~B}-\mathrm{O} 1-\mathrm{H}_{1 \mathrm{~B}}{ }^{\mathrm{viii}}$ & 82.8 \\
\hline $\mathrm{O} 1^{\mathrm{v}}-\mathrm{Fe} 1-\mathrm{O} 1$ & 180.0 & $\mathrm{H} 1 \mathrm{~B}-\mathrm{O} 1-\mathrm{H} 1 \mathrm{~B}^{\mathrm{ix}}$ & 82.8 \\
\hline $\mathrm{C} 1-\mathrm{N} 1-\mathrm{Fe} 1$ & 180.0 & $\mathrm{H} 1 \mathrm{~B}-\mathrm{O} 1-\mathrm{H} 1 \mathrm{~B}^{\mathrm{x}}$ & 138.4 \\
\hline $\mathrm{N} 1-\mathrm{C} 1-\mathrm{Pt} 1$ & 180.0 & $\mathrm{H} 1 \mathrm{~B}^{\mathrm{ix}}-\mathrm{O} 1-\mathrm{H} \mathrm{B}^{\mathrm{x}}$ & 82.8 \\
\hline $\mathrm{Fe} 1-\mathrm{O} 1-\mathrm{H} 1 \mathrm{~A}^{\mathrm{viii}}$ & $115.740(1)$ & $\mathrm{H} 2^{\mathrm{xi}}-\mathrm{O} 2-\mathrm{H} 2^{\mathrm{xii}}$ & 111.0 \\
\hline $\mathrm{Fe} 1-\mathrm{O} 1-\mathrm{H} 1 \mathrm{~A}^{\mathrm{ix}}$ & $115.740(1)$ & $\mathrm{H} 2-\mathrm{O} 2-\mathrm{H}^{\mathrm{xii}}$ & 111.0 \\
\hline $\mathrm{Fe} 1-\mathrm{O} 1-\mathrm{H} 1 \mathrm{~A}$ & 115.7 & $\mathrm{H} 2-\mathrm{O} 2-\mathrm{H} 2^{\mathrm{xi}}$ & 111.0 \\
\hline $\mathrm{Fe} 1-\mathrm{O} 1-\mathrm{H} 1 \mathrm{~A}^{\mathrm{vii}}$ & $115.740(2)$ & $\mathrm{C} 2-\mathrm{O} 2-\mathrm{H} 2$ & 107.9 \\
\hline $\mathrm{Fe} 1-\mathrm{O} 1-\mathrm{H} 1 \mathrm{~B}$ & 110.8 & $\mathrm{C} 2-\mathrm{O} 2-\mathrm{H} 2^{\mathrm{xii}}$ & $107.857(3)$ \\
\hline $\mathrm{Fe} 1-\mathrm{O} 1-\mathrm{H}_{1} \mathrm{~B}^{\mathrm{ix}}$ & 110.8 & $\mathrm{C} 2-\mathrm{O} 2-\mathrm{H} 2^{\mathrm{xi}}$ & $107.857(8)$ \\
\hline $\mathrm{Fe} 1-\mathrm{O} 1-\mathrm{H} \mathrm{B}^{\mathrm{x}}$ & $110.799(2)$ & $\mathrm{O} 2-\mathrm{C} 2-\mathrm{H} 2 \mathrm{~A}$ & 109.5 \\
\hline $\mathrm{Fe} 1-\mathrm{O} 1-\mathrm{H}_{1} \mathrm{~B}^{\mathrm{viii}}$ & 110.8 & $\mathrm{O} 2-\mathrm{C} 2-\mathrm{H} 2 \mathrm{~B}$ & 109.5 \\
\hline $\mathrm{H} 1 \mathrm{~A}^{\mathrm{ix}}-\mathrm{O} 1-\mathrm{H} 1 \mathrm{~A}^{\mathrm{viii}}$ & 79.1 & $\mathrm{O} 2-\mathrm{C} 2-\mathrm{H} 2 \mathrm{C}$ & 109.5 \\
\hline $\mathrm{H} 1 \mathrm{~A}-\mathrm{O} 1-\mathrm{H} 1 \mathrm{~A}^{\mathrm{viii}}$ & 128.5 & $\mathrm{H} 2 \mathrm{~A}-\mathrm{C} 2-\mathrm{H} 2 \mathrm{~B}$ & 109.5 \\
\hline $\mathrm{H} 1 \mathrm{~A}-\mathrm{O} 1-\mathrm{H}_{1} \mathrm{~A}^{\mathrm{vii}}$ & 79.1 & $\mathrm{H} 2 \mathrm{~A}-\mathrm{C} 2-\mathrm{H} 2 \mathrm{C}$ & 109.5 \\
\hline $\mathrm{H} 1 \mathrm{~A}^{\mathrm{ix}}-\mathrm{O} 1-\mathrm{H} 1 \mathrm{~A}^{\mathrm{vii}}$ & 128.5 & $\mathrm{H} 2 \mathrm{~B}-\mathrm{C} 2-\mathrm{H} 2 \mathrm{C}$ & 109.5 \\
\hline $\mathrm{H} 1 \mathrm{~A}^{\mathrm{viii}}-\mathrm{O} 1-\mathrm{H} 1 \mathrm{~A}^{\mathrm{vii}}$ & 79.1 & & \\
\hline
\end{tabular}

Symmetry codes: (i) $-z+1,-y+1, x-1$; (ii) $-x+2,-y+1,-z$; (iii) $z+1, y,-x+1$; (iv) $-y+1,-x+1, z$; (v) $-x+1,-y+1,-z$; (vi) $y, x,-z$; (vii) $-y+1, x, z$; (viii) $y$, $x, z$; (ix) $y,-x+1, z ;$ (x) $-x+1,-y+1, z$; (xi) $-z+1,-x+1, y$; (xii) $-y+1, z,-x+1$.

Hydrogen-bond geometry $\left(\AA,{ }^{\circ}\right)$

\begin{tabular}{lllll}
\hline$D-\mathrm{H} \cdots A$ & $D-\mathrm{H}$ & $\mathrm{H} \cdots A$ & $D \cdots A$ & $D-\mathrm{H} \cdots A$ \\
\hline $\mathrm{O} 1-\mathrm{H} 1 A \cdots \mathrm{O} 2$ & 0.82 & 2.20 & $3.020(18)$ & 175 \\
\hline
\end{tabular}

